\title{
Cosmic acceleration from Abelian symmetry breaking
}

\author{
Gianmassimo Tasinato \\ Institute of Cosmology 83 Gravitation, University of Portsmouth, \\ Dennis Sciama Building, Portsmouth, PO1 3FX, United Kingdom \\ E-mail: gianmassimo.tasinato@port.ac.uk
}

ABSTRACT: We discuss a consistent theory for a self-interacting vector field, breaking an Abelian symmetry in such a way to obtain an interesting behavior for its longitudinal polarization. In an appropriate decoupling limit, the dynamics of the longitudinal mode is controlled by Galileon interactions. The full theory away from the decoupling limit does not propagate ghost modes, and can be investigated in regimes where non-linearities become important. When coupled to gravity, this theory provides a candidate for dark energy, since it admits de Sitter cosmological solutions characterized by a technically natural value for the Hubble parameter. We also consider the homogeneous evolution when, besides the vector, additional matter in the form of perfect fluids is included. We find that the vector can have an important role in characterizing the universe expansion.

Keywords: Cosmology of Theories beyond the SM, Gauge Symmetry, Classical Theories of Gravity

ARXIV EPRINT: 1402.6450 


\section{Contents}

1 Introduction $\quad 1$

2 The set-up $\quad 2$

2.1 Vector field produced by a static source 4

2.2 Relation with scalar Galileons 5

$\begin{array}{ll}2.3 & \text { Coupling to gravity }\end{array}$

$\begin{array}{lll}3 & \text { Applications to cosmology } & 7\end{array}$

$\begin{array}{lll}4 & \text { Discussion } & 10\end{array}$

\section{Introduction}

Imposing a gauge symmetry is a device to remove degrees of freedom. The simplest example is the Abelian U(1) gauge symmetry of electromagnetism. Thanks to this symmetry, the vector associated with a massless photon has two transverse polarizations only, while its longitudinal polarization is absent from the spectrum of dynamical degrees of freedom. Adding a mass term breaks the Abelian gauge symmetry, and makes the longitudinal polarization a dynamical mode. In the limit of vanishing photon mass, the gauge symmetry is recovered in its original form. Neglecting gravity, the longitudinal polarization remains among the available degrees of freedom behaving as a free, massless scalar field that does not interact with the transverse polarization modes.

But the degree of freedom eliminated by imposing an Abelian gauge invariance might not be so undesiderable after all. In some circumstances it can have interesting cosmological applications, as for example to provide a natural candidate for dark energy. Is it possible to break the Abelian gauge symmetry acting on a vector field, in such a way to get a non-trivial theory for its transverse polarization? A motivation for asking this question is an analogy with recent advances on massive gravity. In the dRGT model [1], a proper decoupling limit of vanishing graviton mass leads to a rich theory for the graviton longitudinal polarizations, corresponding to a combination of Galileon Lagrangians [2]. Thanks to its connection with Galileons, dRGT massive gravity is an appealing set-up since it admits cosmological solutions describing accelerating universes in the vacuum [3, 4], exhibits a consistent realization of the Vainshtein screening mechanism [5, 6], and keep quantum corrections under control in the regime of interest $[7,8] .{ }^{1}$ See for example [12-14] for recent general reviews on massive gravity.

\footnotetext{
${ }^{1}$ See however also the works [9-11] that identify problems when applying massive gravity for studying both cosmology and static spherically symmetric configurations.
} 
As we will discuss in this paper, an analogous situation can be obtained in a simpler theory of self-interacting spin one vector fields, with broken Abelian gauge symmetry. When suitable derivative self-interactions are included, the dynamics of the vector longitudinal degree of freedom is non-trivial. In an appropriate decoupling limit, the theory recovers the Abelian gauge invariance, and the dynamics of the vector longitudinal polarization is controlled by a combination of Galileon Lagrangians. The full theory away from the decoupling limit is consistent, in the sense that it does not propagate an additional ghostly fourth mode. The system can be investigated in non-trivial regimes where the effects of non-linear interactions become important. When coupled to gravity, it admits cosmological solutions describing accelerating universes with no need of an additional energy momentum tensor, providing a candidate for dark energy with a technically natural size for the dark energy scale. Moreover, when adding on top of the vector content a combination of perfect fluids with constant equation of state, the resulting cosmological expansion is characterized by a Friedmann equation with peculiar properties and with potentially interesting cosmological consequences. Indeed, we find that the vector can have an important role in characterizing gravitational interactions around cosmological backgrounds, and the cosmological expansion of our universe.

The vector with broken gauge symmetry we are considering is not necessarily the photon. For simplicity, we can regard it as an additional field with no direct couplings to Standard Model particles, although as we will briefly discuss the parameters in our scenario might be accommodated to satisfy the existing bounds. In the past, many scenarios have been considered for modifying General Relativity through the dynamics of vectors, with important cosmological consequences for dark energy and dark matter. The first working models were introduced in the early seventies by Will, Nordtvedt, Hellings [15, 16]; more recently, well studied proposals have been the Einstein-Aether theory [17] and the TeVeS covariantized version of MOND [18]. See [19] for a comprehensive review with a complete list of references to the relevant literature. The novelty of our approach is the emphasis on symmetry arguments for building our theory, so to obtain a compact structure for our Lagrangian that makes explicit connection with Galileons. This fact can allow us to keep our set-up under control in strong coupling regimes, where potentially interesting effects occur.

The paper is organized as follows. We start in section 2 describing how our vector Lagrangian is built, and discussing its physical features including the connection with Galileons. We continue with section 3, investigating applications to cosmology. We conclude in section 4 .

\section{The set-up}

Consider the following vector Lagrangian in Minkowski space (adopting the mostly plus signature), for the moment ignoring any coupling with gravity

$$
\mathcal{L}=-\frac{1}{4} F_{\mu \nu} F^{\mu \nu}+\sum_{i=0}^{3} \mathcal{L}_{(i)}
$$


with $F_{\mu \nu}=\partial_{\mu} A_{\nu}-\partial_{\nu} A_{\mu}$, and $A_{\mu}$ a vector field. The symmetry-breaking Lagrangians $\mathcal{L}_{(i)}$ we consider, besides the usual Proca mass term, are defined in terms of derivative self-interactions of the vector as

$$
\begin{aligned}
\mathcal{L}_{(0)}= & -m^{2} A_{\mu} A^{\mu} \\
\mathcal{L}_{(1)}= & -\beta_{2} A_{\mu} A^{\mu}\left(\partial_{\rho} A^{\rho}\right) \\
\mathcal{L}_{(2)}= & -\frac{\beta_{3}}{m^{2}} A_{\mu} A^{\mu}\left[\left(\partial_{\rho} A^{\rho}\right)\left(\partial_{\nu} A^{\nu}\right)-\left(\partial_{\rho} A^{\nu}\right)\left(\partial^{\rho} A_{\nu}\right)\right] \\
\mathcal{L}_{(3)}= & -\frac{\beta_{4}}{m^{4}} A_{\mu} A^{\mu}\left[-2\left(\partial_{\mu} A^{\mu}\right)^{3}+3\left(\partial_{\mu} A^{\mu}\right)\left(\partial_{\rho} A^{\sigma} \partial^{\rho} A_{\sigma}\right)+3\left(\partial_{\mu} A^{\mu}\right)\left(\partial_{\rho} A^{\sigma} \partial_{\sigma} A^{\rho}\right)\right. \\
& \left.-\partial_{\mu} A^{\nu} \partial_{\nu} A^{\rho} \partial_{\rho} A^{\mu}-3 \partial_{\mu} A^{\nu} \partial_{\nu} A^{\rho} \partial^{\mu} A_{\rho}\right]
\end{aligned}
$$

and break the Abelian gauge symmetry $A_{\mu} \rightarrow A_{\mu}+\partial_{\mu} \xi$. Here, $m$ has dimension of a mass, while the $\beta_{i}$ are dimensionless couplings. In what follow, we will assume a positive $m^{2}$. The suffix $(i)$ in the Lagrangians indicates the number of derivatives in each term. Notice that these interactions do not break Lorentz symmetry, in particular they do not select any preferred frame. The Lagrangians $\mathcal{L}_{(i)}$ are built by the following combinations made with antisymmetric $\epsilon$ tensors in four dimensions

$$
\mathcal{L}_{(i)} \propto A_{\mu} A^{\mu}\left(\epsilon_{\alpha_{1} \ldots \alpha_{i} \gamma_{i+1} \ldots \gamma_{4}} \epsilon^{\beta_{1} \ldots \beta_{i} \gamma_{i+1} \ldots \gamma_{4}} \partial_{\beta_{1}} A^{\alpha_{1}} \ldots \partial_{\beta_{i}} A^{\alpha_{i}}\right)
$$

These derivatives self-interactions are chosen in such a way as to lead to a consistent set-up, in the sense that a fourth 'ghost-mode' cannot be excited. Indeed, due to the antisymmetric properties of the $\epsilon$ tensor, the Lagrangians $\mathcal{L}_{(i)}$ do not contain contributions containing time derivatives of the time component $A_{0}$ of the vector (up to total derivatives): hence the equation of motion for this component is a constraint equation. Let us be more explicit with respect to this point. We decompose the vector in time and spatial components as $A_{\mu}=\left(A_{0}, A_{i}\right)$. Among the various terms associated with the contractions of the indexes in eq. (2.6), we can single out the ones that contain time derivatives of the $A_{0}$ vector component, that result

$$
\mathcal{L}_{(i)} \propto\left(-A_{0}^{2}+A_{i}^{2}\right) \partial_{0} A^{0}\left(\epsilon_{0 \ldots \alpha_{i} \gamma_{i+1} \ldots \gamma_{4}} \epsilon^{0 \ldots \beta_{i} \gamma_{i+1} \ldots \gamma_{4}} \partial_{\beta_{1}} A^{\alpha_{1}} \ldots \partial_{\beta_{i}} A^{\alpha_{i}}\right)+\ldots
$$

where the dots on the right correspond to the remaining terms that do not contain $\partial_{0} A^{0}$.

Integrating by parts eq. (2.7), we find up to total derivatives

$$
\mathcal{L}_{(i)} \propto-2\left(\partial_{0} A_{i}\right) A^{i} A^{0}\left(\epsilon_{0 \ldots \alpha_{i} \gamma_{i+1} \ldots \gamma_{4}} \epsilon^{0 \ldots \beta_{i} \gamma_{i+1} \ldots \gamma_{4}} \partial_{\beta_{1}} A^{\alpha_{1}} \ldots \partial_{\beta_{i}} A^{\alpha_{i}}\right)+\ldots
$$

Notice that, in performing the integration by parts, the time derivative $\partial_{0}$ does not act on the pieces inside the parenthesis of eq. (2.7), since by antisymmetry the $\epsilon$-tensors cancel such contributions. Hence we end with a Lagrangian where time derivatives of $A_{0}$ are absent, and contain only single (time or spatial) derivatives of the other components of the vector: the corresponding equations of motion, then, cannot contain higher derivatives of the fields involved. If such fields have kinetic terms with correct sign (as ensured in Minkowski space by the condition $m^{2}>0$, as we will see in what follows), then the system is free of ghosts. 
On the other hand, the Lagrangians $\mathcal{L}_{i}$ break the Abelian gauge symmetry: the theory contains three dynamical modes, the usual transverse plus the longitudinal polarization of the vector. As we will see, the latter degree of freedom, when $m^{2}>0$, is well behaved. So, we end up with a consistent theory with three healthy modes around Minkowski space.

In what follows, we would like to investigate the interesting dynamics of the vector longitudinal polarization associated with the previous Lagrangians.

\subsection{Vector field produced by a static source}

For simplicity, in this subsection we include (besides the standard kinetic term) the Lagrangians $\mathcal{L}_{(0),(1)}$ only. Hence the Lagrangian on which we now focus our attention is

$$
\mathcal{L}_{T}=-\frac{1}{4} F_{\mu \nu} F^{\mu \nu}-m^{2} A_{\mu} A^{\mu}-\beta A_{\mu} A^{\mu}\left(\partial_{\rho} A^{\rho}\right) .
$$

To gain some initial flavor of the physical effects associated with the non-linear selfcouplings of the vector, let us analyze a static system of a charged density with associated current $J^{\mu}=(\rho, 0,0,0)$, minimally coupled to the vector with a term $A_{\mu} J^{\mu}$ in flat space. We would like to write the equations corresponding to a vector field configuration produced by such a body. We focus on static configurations: $A_{\mu}=A_{\mu}(0, \vec{x})$, and split the vector potential in components as $A_{\mu}=\left(A_{0}, A_{i}\right)$. The equations of motion for the vector degrees of freedom are

$$
\begin{aligned}
-\vec{\nabla}^{2} A_{0} & =\rho-2 m^{2} A_{0}-2 \beta A_{0} \partial_{i} A_{i}, \\
2 m^{2} A_{i} & =\vec{\nabla}^{2} A_{i}-\partial_{i} \partial_{j} A^{j}+\beta \partial_{i}\left(-A_{0}^{2}+A_{j}^{2}\right)-2 \beta A_{i} \partial_{j} A_{j},
\end{aligned}
$$

with $\vec{\nabla}^{2} \equiv \delta_{i j} \partial_{i} \partial_{j}$. The main difference with respect to the gauge invariant (and Proca) cases is that the $\beta$ contribution renders the $A_{0}$ equation dependent on the quantity $\partial_{i} A_{i}$. Taking the divergence of eq. (2.11), we find

$$
2 m^{2} \partial_{i} A_{i}=-\beta \nabla^{2} A_{0}^{2}-2 \beta\left(\partial_{i} A_{i} \partial_{j} A_{j}-\partial_{i} A_{j} \partial_{j} A_{i}\right)
$$

In looking for a static field configuration, we separate the spatial vector components in transverse and longitudinal parts, $A_{i}=A_{i}^{T}+\partial_{i} \chi$ with $\partial_{i} A_{i}^{T}=0$. We focus here on a simplifying Ansatz setting to zero the transverse polarizations $A_{i}^{T}=0$. Hence we end up with the coupled equations for $A_{0}$ and $\chi$

$$
\begin{aligned}
-\vec{\nabla}^{2} A_{0} & =\rho-2 m^{2} A_{0}-2 \beta A_{0} \vec{\nabla}^{2} \chi \\
\vec{\nabla}^{2} \chi & =-\frac{\beta}{2 m^{2}} \vec{\nabla}^{2} A_{0}^{2}-\frac{\beta}{2 m^{2}}\left[\left(\vec{\nabla}^{2} \chi\right)^{2}-\left(\partial_{i} \partial_{j} \chi\right)^{2}\right] .
\end{aligned}
$$

Notice that, although the longitudinal polarization $\chi$ is not directly coupled to the source, nevertheless it 'feels' it via the non-linear term in eq. (2.13). Let us make the further simplifying Ansatz of spherical symmetry, where all the functions depend only on the distance $r$ from the origin, and the previous two equations (2.13)-(2.14), after some manipulations, read

$$
\begin{aligned}
-\frac{d}{d r}\left(r^{2} A_{0}^{\prime}\right) & =r^{2} \rho-2 m^{2} r^{2} A_{0}+2 \beta A_{0} \frac{d}{d r}\left(r^{2} \chi^{\prime}\right), \\
\chi^{\prime} & =\frac{2 \beta}{m^{2}} \frac{\chi^{\prime 2}}{r}+\frac{\beta A_{0} A_{0}^{\prime}}{m^{2}}
\end{aligned}
$$


where a prime indicates derivative along $r$. Eq. (2.16) is a second order algebraic equation for $\chi^{\prime}$, whose solution provides a relation between $\chi$ and $A_{0}$ (we focus only on the branch that decays for large values of $r$ ):

$$
\chi^{\prime}=\frac{m^{2} r}{4 \beta}\left(1-\sqrt{1-\frac{8 \beta^{2} A_{0} A_{0}^{\prime}}{m^{4} r}}\right) .
$$

This relation can be substituted in eq. (2.15) to obtain a non-linear differential equation that govern the behavior of the 'electric field' produced by the source. At large distances from the source, where $A_{0}$ is small, eq. (2.17) can be expressed as

$$
\chi^{\prime} \simeq \frac{\beta}{m^{2}} A_{0} A_{0}^{\prime}
$$

and one finds that both $A_{0}$ and $\chi$ acquire a Yukawa-like suppression (we normalize to unity the charge of the source):

$$
\begin{aligned}
& A_{0} \simeq \frac{e^{-\sqrt{2} m r}}{r}, \\
& \chi \simeq \frac{\beta e^{-2 \sqrt{2} m r}}{2 m^{2} r^{2}} .
\end{aligned}
$$

Notice that $\chi$ decays more rapidly than $A_{0}$. We call $r_{m} \equiv 1 /(\sqrt{2} m)$ the distance at which the Yukawa-like behavior due to the vector mass becomes important in determining the profile for $A_{0}$ : well below this radius, the solution for the vector potential, eq. (2.18), can be approximated by a power-law. In this regime $r \ll r_{m}$, one can identify another characteristic distance, corresponding to the 'strong coupling' scale at which the argument in the square root in eq. (2.17) becomes appreciably different than one: this scale is given by

$$
r_{s} \equiv \frac{\sqrt{\beta}}{m}
$$

By choosing $\beta$ sufficiently small, $r_{s}$ can be made parametrically smaller than $r_{m}$. The regime $r_{s} \ll r \ll r_{m}$ is interesting since the non-linear contributions weighted by $\beta$ in eq. (2.15) can be neglected, as well as the mass term, and the power-law configurations $A_{0} \sim 1 / r, \chi \sim r_{s}^{2} / r^{2}$ are solutions for the equations of motion. It is an intermediate regime in which, although $\chi$ acquires a non-trivial profile due to the non-linear interactions weighted by $\beta$, its effect is too weak to appreciably influence the configuration for $A_{0}$. It would be interesting to numerically investigate the full strong coupling regime $r \ll r_{s}$, in particular to understand whether interesting screening effects on this vector set-up appear, similarly to what happens for the gravitational Vainsthein effect [20].

\subsection{Relation with scalar Galileons}

That some interesting non-linear regime exists nearby a source is suggested by observing that the non-linear equations (2.13)-(2.14) preserve a (spatial) Galileon symmetry in the longitudinal polarization, $\chi \rightarrow \chi+a+b_{i} x_{i}$, and Galileon systems are known to exhibit a screening Vainshtein mechanism [2] in gravitational set-ups. Indeed, our motivation for 
presenting the non-linear coupled equations sourced by a static charge was precisely to point out this fact. We now investigate in more detail how the vector Lagrangian (2.9) is connected with Galileons. We adopt the Stückelberg formalism, trading everywhere $A_{\mu}$ for $A_{\mu}+1 /(\sqrt{2} m) \partial_{\mu} \phi$ : the resulting Lagrangian is invariant under the gauge symmetry $A_{\mu} \rightarrow A_{\mu}-\partial_{\mu} \xi, \phi \rightarrow \phi+\sqrt{2} m \xi$. The scalar field $\phi$ plays the same physical role as that of the longitudinal vector polarization. The use of the Stückelberg approach renders clearer the interactions among the different degrees of freedom. The total Lagrangian reads, assuming $m^{2}>0$ to avoid ghost instabilities,

$$
\begin{aligned}
\mathcal{L}_{T}= & -\frac{1}{4} F_{\mu \nu} F^{\mu \nu}-\frac{1}{2}\left(\sqrt{2} m A_{\mu}+\partial_{\mu} \phi\right)\left(\sqrt{2} m A^{\mu}+\partial^{\mu} \phi\right) \\
& -\frac{\beta}{\sqrt{8} m^{3}}\left(\sqrt{2} m A_{\mu}+\partial_{\mu} \phi\right)\left(\sqrt{2} m A^{\mu}+\partial^{\mu} \phi\right)\left(\sqrt{2} m \partial_{\nu} A^{\nu}+\partial_{\nu} \partial^{\nu} \phi\right) .
\end{aligned}
$$

To isolate the (self-)interactions of the Stückelberg field $\phi$ we take the 'decoupling' limit

$$
m \rightarrow 0, \beta \rightarrow 0, \quad \frac{\beta}{m^{3}}=\text { fixed }=\frac{\sqrt{2}}{\Lambda_{G}^{3}},
$$

leading to

$$
\mathcal{L}_{\text {dec }}=-\frac{1}{4} F_{\mu \nu} F^{\mu \nu}-\frac{1}{2} \partial_{\mu} \phi \partial^{\mu} \phi-\frac{1}{2 \Lambda_{G}^{3}}\left(\partial_{\mu} \phi \partial^{\mu} \phi\right) \partial_{\nu} \partial^{\nu} \phi
$$

The result of taking such a decoupling limit is a theory with two different symmetries: ${ }^{2}$ a free vector Lagrangian that satisfies the Abelian gauge symmetry, plus a cubic Galileon scalar Lagrangian controlled by the strong coupling scale $\Lambda_{G}$, and respecting a Galileon symmetry $\pi \rightarrow \pi+b+a_{\mu} x^{\mu}$. This feature makes stable the size of the parameters $m$ and $\beta$, since keeping them small is technically natural in the 't Hooft sense [21]. It would also be interesting to analyze in detail the issue of quantum corrections to this set-up. In particular, to try to understand whether additional operators - that would spoil the structure of our Lagrangian - can be kept under control when working in some strong or intermediate coupling regimes, in analogy with what happens for Galileons or massive gravity $[7,8]$. Related to this, it would be interesting to understand whether conformal versions of this vector Lagrangian can be constructed, using for example the methods of [22], to find relations with conformal Galileon theories [2].

Moreover, the connection we found with Galileons provides another perspective on why the theory under consideration is consistent (ghost free) around Minkowski space, and promises to lead to interesting cosmological applications as accelerating configurations.

\subsection{Coupling to gravity}

Coupling our theory to gravity presents the very same issues one meets in the covariantization of scalar Galileon theories. In order not to propagate ghosts, we require that our set-up does not lead to derivatives higher than two in the equations of motion for vector and

\footnotetext{
${ }^{2}$ Analogous arguments straightforwardly apply also to the complete set of interactions $\mathcal{L}_{(i)}$ in eq. (2.1), leading to higher order scalar Galileon Lagrangians.
} 
gravitational degrees of freedom. Applying for example the approach developed in [23, 24], one finds a consistent covariantization of the Lagrangian densities $\mathcal{L}_{(1)}, \mathcal{L}_{(2)}$ :

$$
\begin{aligned}
& \mathcal{L}_{(1)}^{\text {cov }}=-\beta_{1} A_{\mu} A^{\mu}\left(\nabla_{\rho} A^{\rho}\right), \\
& \mathcal{L}_{(2)}^{\text {cov }}=-\frac{\beta_{2}}{m^{2}} A_{\mu} A^{\mu}\left[\left(\nabla_{\rho} A^{\rho}\right)\left(\nabla_{\nu} A^{\nu}\right)-\left(\nabla_{\rho} A^{\nu}\right)\left(\nabla^{\rho} A_{\nu}\right)-\frac{1}{4} R A_{\sigma} A^{\sigma}\right],
\end{aligned}
$$

with $\nabla_{\mu}$ the usual covariant derivative in curved space, and $R$ is the Ricci scalar. Notice that the vectors couple non-minimally to gravity, thanks to the coupling with the Ricci scalar in eq. (2.25). For our purposes, we will not need to covariantize $\mathcal{L}_{(3)}$ : this is left for future work. It is simple to check that in an appropriate decoupling limit (as discussed in subsection 2.2) the previous formulae reduce to the covariantized cubic and quartic scalar Galileon Lagrangians. It would be interesting to analyze whether the vector interactions can contribute to a gravitational Vainshtein mechanism around a spherically symmetric source, as investigated for a scalar-vector set-up in [25].

Armed with these results, we will now focus on the action

$$
\mathcal{S}=\int d^{4} x \sqrt{-g}\left[\frac{M_{\mathrm{Pl}}^{2}}{2} R-\frac{1}{4} F_{\mu \nu} F^{\mu \nu}-m^{2} A_{\mu} A^{\mu}+\mathcal{L}_{(1)}^{\text {cov }}+\mathcal{L}_{(2)}^{\text {cov }}\right]
$$

with the aim to study its cosmological implications.

\section{Applications to cosmology}

We consider a homogeneous FRW metric with flat spatial curvature

$$
d s^{2}=-d t^{2}+a^{2}(t) \delta^{i j} d x_{i} d x_{j}
$$

with $a$ the scale factor, and $H=\dot{a} / a$ the corresponding Hubble parameter. The vector potential is $A_{\mu}=\left(A_{0}, A_{i}\right)$. The spatial vector components are decomposed in $A_{i}=A_{i}^{T}+\partial_{i} \chi$ with $\partial_{i} A_{i}^{T}=0$. We investigate homogeneous configurations. We consider a background vector profile with only the time-component turned on: $A_{\mu}=\left(A_{0}(t), 0,0,0\right)$. We avoid to turn on spatial components for the vector to avoid anisotropies and the corresponding generic instabilities pointed out in [26]. The equation of motion for $A_{0}$ is a constraint equation, since the Lagrangian does not depend on time derivatives of $A_{0}$, and reads

$$
A_{0}\left(m^{2}-3 \beta_{1} A_{0} H+9 \frac{\beta_{2}}{m^{2}} A_{0}^{2} H^{2}\right)=0 .
$$

We can identify various branches of solutions: one is the trivial $A_{0}=0$, while the most interesting ones for us are

$$
\begin{aligned}
A_{0}^{ \pm}(t) & =\frac{\beta_{1} \pm \sqrt{\beta_{1}^{2}-4 \beta_{2}}}{6 \beta_{2}} \frac{m^{2}}{H(t)}, \\
& =\frac{c_{ \pm} m^{2}}{H(t)} .
\end{aligned}
$$


These branches require $\beta_{1}^{2} \geq 4 \beta_{2}$ to have a real square root. In the second line we defined the dimensionless parameters $c_{ \pm}$built in terms of $\beta_{1}, \beta_{2}$. From now on, for definiteness, we will focus on the case $\beta_{1} \geq 0, \beta_{2} \geq 0$. Using the non-trivial solutions (3.3) for $A_{0}$, one finds that the content of the energy momentum tensor has a perfect fluid structure, with vector energy density and pressure given by

$$
\begin{aligned}
& \rho_{V}=\frac{c_{ \pm}^{2}\left(9 \beta_{2} c_{ \pm}^{2}-2\right) m^{6}}{2 H^{2}}, \\
& p_{V}=\frac{c_{ \pm}^{2}\left(2-9 \beta_{2} c_{ \pm}^{2}\right) m^{6}}{2 H^{2}}+\frac{c_{ \pm}^{3}\left(9 \beta_{2} c_{ \pm}-2 \beta_{1}\right) \dot{H}}{H^{4}} .
\end{aligned}
$$

It is simple to show that, in order to have a positive vector energy density, $\rho_{V} \geq 0$, one has to focus on the positive branch of solutions in eq. (3.2), that require a non-vanishing $\beta_{2}$. The Friedmann equation reads

$$
H^{2}=\frac{c_{ \pm}^{2}\left(9 \beta_{2} c_{ \pm}^{2}-2\right) m^{6}}{6 H^{2} M_{\mathrm{Pl}}^{2}}
$$

that is solved for a constant Hubble parameter. A real solution for the scale factor can be found focussing on the positive branch of eq. (3.2), where the (square of the) Hubble parameter results

$$
H^{2}=\left(\frac{c_{+}}{\sqrt{6}} \sqrt{9 \beta_{2} c_{+}^{2}-2}\right) \frac{m^{3}}{M_{\mathrm{Pl}}}
$$

and is well defined when $\beta_{1}^{2}>9 \beta_{2} / 2$, a condition that we will impose from now on. The overall dimensionless coefficient in front of the right hand side of the previous equation call it $c_{\beta}$ - simplifies in the small $\beta_{2}$ limit, reducing to $c_{\beta} \simeq \beta_{1}^{2} /\left(108 \beta_{2}^{3}\right)^{1 / 2}$.

Hence, the dynamics associated with the new vector interactions is able to drive cosmological acceleration with a constant (de Sitter) equation of state. At the background level, such cosmological acceleration is identical to the one driven by a positive 'cosmological constant' of size

$$
\Lambda_{V}^{4}=6 c_{\beta} m^{3} M_{\mathrm{Pl}}
$$

where the quantity $\Lambda_{V}$ has the dimension of a mass, and allows us to write more concisely $H^{2}=\Lambda_{V}^{4} /\left(6 M_{\mathrm{Pl}}^{2}\right)$. In order to be able to drive a de Sitter expansion with the current value for the Hubble parameter, the mass parameter $m$ should be chosen to be of order

$$
m \simeq c_{\beta}^{-1 / 3} 10^{-13} \mathrm{eV} .
$$

The current limit on the photon mass is $m_{\gamma} \leq 10^{-18} \mathrm{eV}$ [27], that could be satisfied by taking a sufficiently small value for $c_{\beta}$. Small values for our parameters are technically natural in the 't Hooft sense, since as sending $m$ (and the $\beta_{i}$ ) to zero one recovers Abelian and Galileon symmetries. Hence, although we are keeping our discussion completely general, one might think to use the photon itself as the self-interacting vector we are investigating. Let us point out that the non-linear vector interactions we are analyzing, with their associated strong coupling effects, can considerably affect the existing bounds: see [28] for a critical discussion on photon mass limits. 
It would also be interesting to study in detail the dynamics of cosmological perturbations around the time dependent configurations we have presented. We leave this task for future work, but let us mention that we checked that, after including the contributions from the homogeneous background, the effective mass parameter for the transverse vector fluctuations $A_{i}^{T}$ does maintain the correct sign around this cosmological solution.

Let us investigate a bit further the background homogeneous cosmology in our set-up. We will see that vectors can have an interesting role in characterizing the cosmological evolution. On top of the vector content previously analyzed, we include additional matter content in the form of perfect fluids with constant equation of state, with total energy density $\rho$, for simplicity not directly coupled to the vector. The first Friedmann equation now reads

$$
H^{2}(\tau)=\frac{\rho}{3 M_{\mathrm{Pl}}^{2}}+\frac{\Lambda_{V}^{8}}{36 H^{2}(\tau) M_{\mathrm{Pl}}^{4}}
$$

with $\Lambda_{V}^{4}$ the effective cosmological constant induced by the vector, as defined in equation (3.8). The second contribution is peculiar, since it contains an $H^{2}$ in the denominator. Eq. (3.10) can be solved expressing the Hubble parameter in terms of the remaining quantities: the branch of solutions corresponding to a real $H$ is

$$
H^{2}=\frac{\rho+\sqrt{\rho^{2}+\Lambda_{V}^{8}}}{6 M_{\mathrm{Pl}}^{2}} .
$$

Such 'Friedmann-like' equation has a non-standard structure, due to the square root in the right hand side. Interestingly, it admits solutions also for a negative energy density $\rho$ (for example, a $\rho$ dominated by a negative bare cosmological constant) in absence of spatial curvature.

The standard form of the Friedmann equation - in absence of a cosmological constant — is obtained in the limit $\rho \gg \Lambda_{V}^{4}$. In the opposite limit, $\rho \ll \Lambda_{V}^{4}$, we expand (3.11) obtaining

$$
H^{2}=\frac{\rho}{6 M_{\mathrm{Pl}}^{2}}+\frac{\Lambda_{V}^{4}}{6 M_{P l^{2}}}+\frac{\rho^{2}}{12 \Lambda_{V}^{4} M_{\mathrm{Pl}}^{2}}+\ldots
$$

The linear term in $\rho$ differs from the standard form for the Friedmann equation due to the factor of two in the denominator. This suggests that, in this small $\rho$ limit, the effective Newton constant in this cosmological background is half the one in Minkowski space, in other words $M_{\mathrm{Pl}}^{\text {cosm }}=\sqrt{2} M_{\mathrm{Pl}}^{\mathrm{Mink}}$. (We checked that the same behavior occurs for the second Friedmann equation, governing the second time derivative of the scale factor.) Hence, vector degrees of freedom play a relevant role in characterizing gravitational interactions and cosmological evolution around non-trivial backgrounds, since they 'renormalize' the value of the Newton constant. This fact could also be argued from the structure of our covariantized action in equation (2.25), where we learn that vectors are non-minimally coupled with the Ricci scalar. This implies that the dimensionful coefficient in front of the Ricci scalar in the action - that sets the strength of gravitational interactions - can depend on the vector background. It would be very interesting to directly calculate the gravitational force between test bodies in these cosmological configurations, to understand more explicitly the role of vectors in determining the gravitational force. 


\section{Discussion}

In this work we discussed a consistent theory for a self-interacting vector field that breaks an Abelian symmetry, in such a way to obtain an interesting dynamics for the vector longitudinal polarization. In an appropriate decoupling limit, the dynamics of the longitudinal scalar mode is controlled by Galileon Lagrangians. The full theory away of the decoupling limit is consistent in the sense that it does not propagate a ghostly fourth mode. The system can be investigated in non-trivial regimes where the effects of non-linear interactions become important. When coupled with gravity it admits a de Sitter branch of cosmological solutions characterized by a technically natural value for the Hubble parameter. We studied the homogeneous cosmological evolution when additional matter in the form of perfect fluids is included in the energy momentum tensor. The resulting cosmological expansion is characterized by a Friedmann equation with peculiar properties and with potentially interesting cosmological consequences. Indeed, we found that the vector can have an important role in characterizing gravitational interactions around cosmological backgrounds, and the cosmological expansion of our universe.

As mentioned above, the non-linear self-interactions for the transverse vector polarizations are controlled by Galileon combinations; hence, strong coupling effects can play a role in physically interesting situations. The relation with Galileon and Abelian symmetries in appropriate limits renders the theory technically natural, allowing to keep the size of the available parameters under control. It would be interesting to further explore our theory in non-linear regimes to understand whether the particular structure we have chosen for our Lagrangian remains valid when quantum corrections are taken into account. Also, on a more phenomenological side, it will be important to investigate in more details the accelerating cosmological configurations we have determined, in particular the stability of fluctuations around them.

While in this work we did not specify the microscopic nature of the vector, it will be interesting to explore more in detail whether the photon can play its role. We have explained that current photon mass limits can be satisfied by a suitable and technically natural choice of the available parameters. On the other hand, it is very likely that the non-linear interactions we have analyzed considerably affect the existing bounds. Besides cosmology, it would also be interesting to investigate whether our interactions can be obtained via a Higgs mechanism, and whether they can be realized in some specific condensed matter physics set-up where Abelian symmetries are spontaneously broken.

\section{Acknowledgments}

It is a pleasure to thank Marco Crisostomi, Matthew Hull, Kazuya Koyama, Gustavo Niz, Ivonne Zavala for useful comments on the draft, and STFC for financial support through the grant ST/H005498/1.

Open Access. This article is distributed under the terms of the Creative Commons Attribution License (CC-BY 4.0), which permits any use, distribution and reproduction in any medium, provided the original author(s) and source are credited. 


\section{References}

[1] C. de Rham, G. Gabadadze and A.J. Tolley, Resummation of Massive Gravity, Phys. Rev. Lett. 106 (2011) 231101 [arXiv:1011.1232] [INSPIRE].

[2] A. Nicolis, R. Rattazzi and E. Trincherini, The Galileon as a local modification of gravity, Phys. Rev. D 79 (2009) 064036 [arXiv:0811.2197] [InSPIRE].

[3] C. de Rham, G. Gabadadze, L. Heisenberg and D. Pirtskhalava, Cosmic Acceleration and the Helicity-0 Graviton, Phys. Rev. D 83 (2011) 103516 [arXiv:1010.1780] [InSPIRE].

[4] G. D'Amico et al., Massive Cosmologies, Phys. Rev. D 84 (2011) 124046 [arXiv:1108.5231] [INSPIRE].

[5] K. Koyama, G. Niz and G. Tasinato, Strong interactions and exact solutions in non-linear massive gravity, Phys. Rev. D 84 (2011) 064033 [arXiv:1104.2143] [INSPIRE].

[6] K. Koyama, G. Niz and G. Tasinato, Analytic solutions in non-linear massive gravity, Phys. Rev. Lett. 107 (2011) 131101 [arXiv:1103.4708] [INSPIRE].

[7] A. Nicolis and R. Rattazzi, Classical and quantum consistency of the DGP model, JHEP 06 (2004) 059 [hep-th/0404159] [INSPIRE].

[8] C. de Rham, G. Gabadadze, L. Heisenberg and D. Pirtskhalava, Nonrenormalization and naturalness in a class of scalar-tensor theories, Phys. Rev. D 87 (2013) 085017 [arXiv: 1212.4128] [INSPIRE].

[9] K. Koyama, G. Niz and G. Tasinato, The Self-Accelerating Universe with Vectors in Massive Gravity, JHEP 12 (2011) 065 [arXiv:1110.2618] [INSPIRE].

[10] A.E. Gumrukcuoglu, C. Lin and S. Mukohyama, Cosmological perturbations of self-accelerating universe in nonlinear massive gravity, JCAP 03 (2012) 006 [arXiv:1111.4107] [INSPIRE].

[11] C. Burrage, N. Kaloper and A. Padilla, Strong Coupling and Bounds on the Spin-2 Mass in Massive Gravity, Phys. Rev. Lett. 111 (2013) 021802 [arXiv:1211.6001] [INSPIRE].

[12] K. Hinterbichler, Theoretical Aspects of Massive Gravity, Rev. Mod. Phys. 84 (2012) 671 [arXiv: 1105.3735] [INSPIRE].

[13] G. Tasinato, K. Koyama and G. Niz, Exact Solutions in Massive Gravity, Class. Quant. Grav. 30 (2013) 184002 [arXiv: 1304.0601] [INSPIRE].

[14] C. de Rham, Massive Gravity, arXiv:1401.4173 [INSPIRE].

[15] C.M. Will and K. Nordtvedt Jr., Conservation Laws and Preferred Frames in Relativistic Gravity. I. Preferred-Frame Theories and an Extended PPN Formalism, Astrophys. J. 177 (1972) 757 [INSPIRE].

[16] R.W. Hellings and K. Nordtvedt, Vector-Metric Theory of Gravity, Phys. Rev. D 7 (1973) 3593 [InSPIRE].

[17] T. Jacobson and D. Mattingly, Gravity with a dynamical preferred frame, Phys. Rev. D 64 (2001) 024028 [gr-qc/0007031] [INSPIRE].

[18] J.D. Bekenstein, Relativistic gravitation theory for the MOND paradigm, Phys. Rev. D 70 (2004) 083509 [Erratum ibid. D 71 (2005) 069901] [astro-ph/0403694] [INSPIRE]. 
[19] T. Clifton, P.G. Ferreira, A. Padilla and C. Skordis, Modified Gravity and Cosmology, Phys. Rept. 513 (2012) 1 [arXiv:1106.2476] [InSPIRE].

[20] A. Vainshtein, To the problem of nonvanishing gravitation mass, Phys. Lett. B 39 (1972) 393 [INSPIRE].

[21] G. 't Hooft, Naturalness, chiral symmetry, and spontaneous chiral symmetry breaking, NATO Adv. Study Inst. B 59 (1980) 135.

[22] G. Tasinato, Conformal couplings of Galileons to other degrees of freedom, JHEP 11 (2013) 196 [arXiv:1310.0034] [INSPIRE].

[23] C. Deffayet, G. Esposito-Farese and A. Vikman, Covariant Galileon, Phys. Rev. D 79 (2009) 084003 [arXiv:0901.1314] [INSPIRE].

[24] C. Deffayet, S. Deser and G. Esposito-Farese, Generalized Galileons: all scalar models whose curved background extensions maintain second-order field equations and stress-tensors, Phys. Rev. D 80 (2009) 064015 [arXiv:0906.1967] [InSPIRE].

[25] G. Tasinato, K. Koyama and N. Khosravi, The role of vector fields in modified gravity scenarios, JCAP 11 (2013) 037 [arXiv: 1307.0077] [INSPIRE].

[26] B. Himmetoglu, C.R. Contaldi and M. Peloso, Instability of anisotropic cosmological solutions supported by vector fields, Phys. Rev. Lett. 102 (2009) 111301 [arXiv:0809.2779] [inSPIRE].

[27] Particle Data Group collaboration, J. Beringer et al., Review of Particle Physics (RPP), Phys. Rev. D 86 (2012) 010001 [inSPIRE].

[28] A.S. Goldhaber and M.M. Nieto, Photon and Graviton Mass Limits, Rev. Mod. Phys. 82 (2010) 939 [arXiv:0809.1003] [InSPIRE]. 